\title{
Cambios en la vegetación durante el Holoceno en la región Norte del Iberá, Corrientes, Argentina
}

Lionel Fernández Pacella ${ }^{1}$, Silvina Garralla ${ }^{2}$ \& Luisa Anzótegui ${ }^{3}$

1. Universidad Nacional del Nordeste, Centro de Ecología Aplicada del Litoral, CONICET, Ruta 5, km 2.5, CP 3400Corrientes, Argentina; lionelpacella@yahoo.com.ar

2. Universidad Nacional del Nordeste, Centro de Ecología Aplicada del Litoral, CONICET, Ruta 5, km 2.5, CP 3400Corrientes, Argentina.

3. Facultad de Ciencias Exactas y Naturales y Agrimensura, Centro de Ecología Aplicada del Litoral, CONICET, Ruta 5, km 2.5, CP 3400- Corrientes, Argentina.

Recibido 30-XI-2009. Corregido 06-VIII-2010. Aceptado 01-IX-2010.

\begin{abstract}
Vegetation changes during the Holocene in the North Iberá, Corrientes, Argentina. Wetlands are very important sites for palynological studies, since they represent one of the most suitable environments for fossil pollen preservation. The aim of this work was to determine, by palynological analysis of lacustrine sediments, the vegetal communities and the predominant environment during the Holocene in NW of Iberá. Two lagoons were studied: San Sebastián and San Juan Poriahú. Sediment samples were obtained with witness using a "Levingstone square-rod sampler", processed with Faegri e Iversen techniques and dated with $\mathrm{C}^{14}$. The palynological graphs were divided in zones using the Tilia program. The palynological analysis allowed visualizing diverse changes in the vegetation: from $6140 \pm 50$ to $5170 \pm 100$ a. C., the NW of Iberá was characterized by marsh-herbaceous vegetation and arboreal vegetation typical of dry vegetation. From $5170 \pm 100$ to $3460 \pm 60$ a. C., a decrease in the species frequency, typical of wet environments, is produced, and the clogging of the waterbody, from $3460 \pm 60$ a. C. onwards, while continuing the dominance of herbaceous vegetation typical of these environments, the arboreal pollen, indicates the beginning of a hygrophilous forest development. Rev. Biol. Trop. 59 (1): 103-112. Epub 2011 March 01.
\end{abstract}

Key words: wetlands, palynomorphs, lacustrine sediments, vegetal communities, environment.

El Iberá constituye un sistema hídrico alimentado fundamentalmente por lluvias (1 200 a $1500 \mathrm{~mm}$ año). El clima de la zona es subtropical húmedo, con influencia oceánica dado que el anticiclón del Atlántico Sur moviliza masas de vientos húmedos y cálidos por el nordeste de Argentina (Neiff 1997).

La cuenca iberiana se desarrolló, en el margen occidental, sobre una base de arenas. Su origen, según Popolizio (1970), es de tipo pseudokárstico y posterior hundimiento de las capas de arena suprayacentes, mientras que Iriondo (1981) sugiere que serían más bien de origen eólico, debido a la acción del viento durante los períodos más secos. En esta margen se asientan numerosas lagunas de variados tamaños cuyos fondos están cubiertos por sedimentos arenosos, correspondiente al Plioceno Superior - Pleistoceno Inferior.

En las lagunas, la estructura y organización de la vegetación actual acuática es muy compleja, abundan las praderas sumergidas de Cabomba australis Speg., Egeria naias Planch., Utricularia foliosa Kamiénski entre otras plantas, asentadas en un sedimento fino y rico en materia orgánica que puede extenderse por cientos de metros. Por encima se encuentran los embalsados (sustrato o suelo flotante) constituidos por una trabazón de raíces, a lo que se agregan detritos inorgánicos y plantas flotantes, los que están conformados por plantas anfibias (Panicum grumosum Nees, Thypha sp. L., Thalia multiflora Sw. y Zizaniopsis sp. Döll y Asch.) que se asocian con otras de 
considerable porte e incluso forman pequeños bosques, las especies más comunes son Sapium haematospermum Müll. Arg., Ocotea acutifolia (Nees) Mez o Croton urucurana Baillon entre otras (Canevari et al. 1998). Con respecto a la vegetación regional, esta se caracteriza (al NO del Iberá) por la presencia del bosque higrófilo, constituido por tres estratos: 1) arbóreo: compuesto principalmente por Tabebuia heptaphylla Gómez, Peltophorum dubium (Vogel) Benth. y Nectandra angustifolia Rol. ex Rottb.(entre otros); 2) arbustivo: cuyos elementos más importantes son: Brunfelsia australis Plum. ex L., Urera baccifera (L.) Gaud., Byttneria urticufolia L. y Ephedra tweediana L.; 3) por último se encuentra el estrato herbáceo integrado por: Oplismenus hirtellus Merr., Chaptalia nutans Vent., Petiveria alliacea L. y Scleria melaleuca Bergius (entre otros). Al SO del Iberá, la vegetación, se caracteriza por la presencia de bosques abiertos con predominancia de Prosopis affinis Sprengel, Prosopis nigra Griseb. y Acacia caven (Molina) Molina (Arbo $\&$ Tressens 2002).

Por los numerosos intereses (económicos, conservacionistas, políticos, entre otros) que despierta este sistema en Argentina y en Latinoamérica en general, ha sido objeto de diversos estudios que han involucrado su fauna, vegetación actual, geomorfología y ecología (Neiff 1977, 1981a, 1981b, Poi de Neiff 1981, Neiff 1982, Cuadrado \& Neiff 1993, Carnevali 1994, Neiff 1997, Canevari et al. 1998, Arbo \& Tressens 2002); pero desde el punto de vista palinológico, Cuadrado \& Neiff (1993), y posteriormente Fernández Pacella (2005), analizaron algunos sedimentos de embalsados y del fondo lagunar, sentando así las bases para futuros análisis de este tipo. Otros antecedentes de estudios palinológicos en el noreste de Argentina fue el realizado por Garralla (1998) en el Norte de la provincia de Santa Fe, señalando el tipo de vegetación y las condiciones paleoambientales predominantes durante el Holoceno Superior.

En la provincia de Corrientes, los sistemas de esteros y lagunas del Iberá, abarcan aproximadamente $12000 \mathrm{~km}^{2}$ de superficie y es considerado uno de los humedales más importantes de Latinoamérica (Neiff 1997). Dado que los humedales cumplen un importante papel en la regulación del clima regional y son uno de los ambientes terrestres más aptos para la preservación del polen fósil, se analizaron palinológicamente los sedimentos de fondo de dos lagunas. El objetivo del presente trabajo es determinar a partir del análisis palinológico de los sedimentos lacustres, las comunidades vegetales y el ambiente predominante durante el Holoceno en la región NO del sistema Iberá.

\section{MATERIALES Y MÉTODOS}

El centro aproximado de este sistema se ubica en las coordenadas $28^{\circ} 36^{\prime} 0^{\prime \prime} \mathrm{S}-5^{\circ} 49^{\prime} 0^{\prime \prime}$ W. (Fig. 1), y se analizaron palinológicamente los sedimentos de fondo de las lagunas San Sebastián (2752'58.10" S - 57²0'34.03” W)

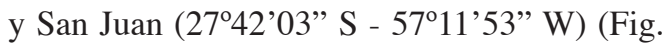
2), ubicadas en el departamento de San Miguel en la provincia de Corrientes. Las mismas son circulares, poco profundas (hasta 6 metros), de fácil acceso y forman parte de una secuencia de lagunas ubicadas a lo largo del límite noroeste del sistema. Se asientan sobre una extensa

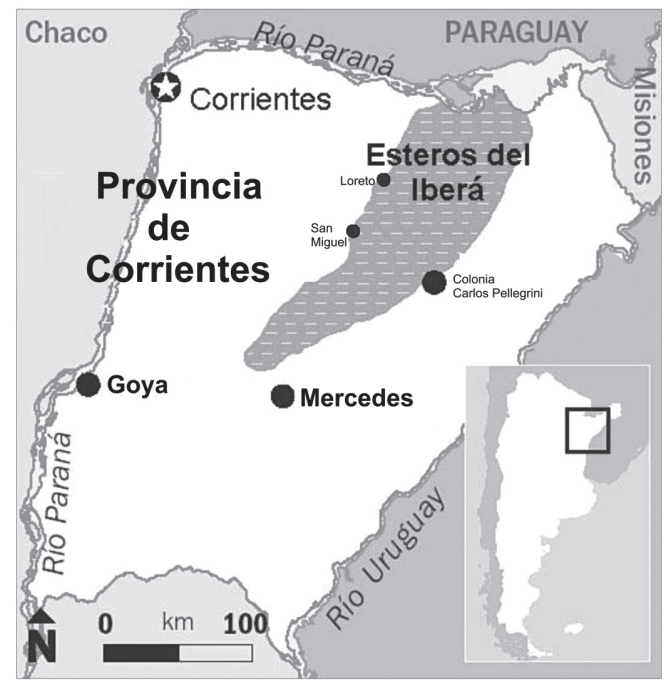

Fig. 1. Esteros del Iberá en la Provincia de Corrientes.

Fig. 1. Iberá Estuaries in the Province of Corrientes. 


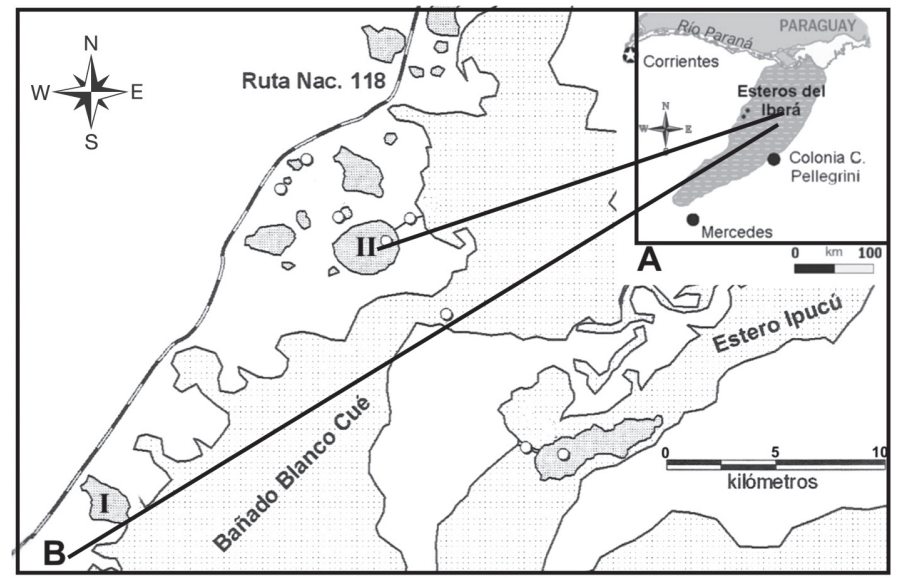

Fig. 2. A. Lagunas estudiadas, en los Esteros del Iberá. B. Detalle de secuencia de las lagunas ubicadas al noroeste del sistema; I. Laguna San Sebastián; II. Laguna San Juan. (Extraído y modificado de Casciotta et al. 2005).

Fig. 2. A. The studied lagoons, in the Iberá matting. B. Sequence detail of the lagoons located of NW of the system; I. Lagoon San Sebastián; II. Lagoon San Juan. (Extracted and modified from Casciotta et al. 2005).

lomada arenosa perteneciente a la Formación Ituzaingó (compuesto por arenisca y arcilla), depositada durante el Pleistoceno.

Los sedimentos de las lagunas estudiadas, fueron obtenidos del centro de las mismas, con un sacatestigo "Levingstone square-rod sampler", hasta los $24 \mathrm{~cm}$ en la laguna San Sebastián y hasta los $75 \mathrm{~cm}$ de profundidad en San Juan Poriahú; la diferencia en longitud de los perfiles se debe a que el sacatestigo solo extrae sedimento orgánico y arcilla y no arena; por lo tanto esta capa de arena constituye el límite de extracción de sedimento, encontrándose a los $24 \mathrm{~cm}$ de profundidad en San Sebastián y a $\operatorname{los} 75 \mathrm{~cm}$ en San Juan. En ambas lagunas, los sedimentos extraídos son de color castaño oscuro a negro. El testigo correspondiente a la laguna San Sebastián fue muestreado cada $2 \mathrm{~cm}$ obteniéndose un total de 12 muestras, y en la laguna San Juan se muestreó cada $4 \mathrm{~cm}$ (incluyendo el nivel 0) y se tomaron 20 muestras, esta diferencia de muestreo se debe a la textura del sedimento. La extracción de polen y esporas se realizó por el método estándar de Faegri \& Iversen (1989) que consistieron en: defloculación de arcillas con hexametafosfato de sodio al 10\%, eliminación de ácidos húmicos con hidróxido de sodio al 5\%, eliminación de los carbonatos con unas gotas de $\mathrm{HCl}$ al $10 \%$, separación de la materia orgánica de la inorgánica con líquidos pesados $\left(\mathrm{ZnCl}_{2}\right)$ y eliminación de los silicatos con HF. Con el fin de calcular la concentración polínica de los sedimentos a cada muestra se le agregó 5 tabletas de Lycopodium sp. El montaje de las láminas se realizó en glicerina-gelatina pura. El recuento de palinomorfos se efectuó con un microscopio OLYMPUS BH2. Se contaron como mínimo 300 palinomorfos por nivel muestreado. Los preparados palinológicos se hallan depositados en la colección PMP-CTES de la Facultad de Ciencias Exactas y Naturales y Agrimensura de la Universidad Nacional del Nordeste.

La materia orgánica, hallada en los sedimentos de ambas lagunas, fueron datados mediante la técnica radiométrica con $\mathrm{C}^{14}$, en los laboratorios QUADRU (Quaternary Dating Research Unit), en Pretoria, Sudáfrica y en Beta Analytic Laboratory (Miami, Florida) (Cuadro 1).

Los diagramas palinológicos se dividieron en "zonas" mediante el análisis de 
CUADRO 1

Dataciones con C14

\begin{tabular}{lccccc}
\multicolumn{1}{c}{ Lagunas } & $\begin{array}{c}\text { Niveles } \\
\text { datados }(\mathrm{cm})\end{array}$ & $\mathrm{C}^{13}$ & $\begin{array}{c}\text { Edad C } \\
(\mathrm{Años} A P)\end{array}$ & Material datado & Laboratorio N $^{\circ}$ \\
San Sebastián & 9 & -18.8 & $5170 \pm 100$ & Materia orgánica & 249278 (Beta Analytic) \\
San Sebastián & 24 & -17.1 & $6140 \pm 50$ & Materia orgánica & 259457 (Beta Analytic) \\
San Juan & 15 & -18.4 & $3450 \pm 40$ & Materia orgánica & 249277 (Beta Analytic) \\
San Juan & 75 & -17.8 & $3460 \pm 60$ & Materia orgánica & 9200 (QUADRU)
\end{tabular}

agrupamiento en modo Q, utilizando la Distancia Euclidiana. Para ello se utilizó el programa Tilia. La identificación y determinación de los palinomorfos a nivel de familia, género o especie se realizó mediante comparación y con la ayuda de la bibliografía específica y material de referencia, además para la suma polínica se excluyeron pteridófitas, algas y hongos. Los porcentajes mencionados, para cada taxón, representan los valores máximos registrados para cada zona.

\section{RESULTADOS}

Los resultados del análisis palinológico se presentan en diagramas porcentuales palinológicos y valores de concentración total (granos/g) (Figs. 3 y 4).

Laguna San Sebastián: Se diferencian dos zonas (A y B) (Fig. 3)

Zona A: Desde los $24 \mathrm{~cm}$ hasta los $10 \mathrm{~cm}$ de profundidad. En esta zona, el polen arbóreo esta representado por Arecastrum sp. (7\%), Astronium balansae Engl. (6\%), y Tecoma stans (L.) Juss. Kunth (2\%), mientras que los de Prosopis sp., Myrtaceae y Chrysophyllum sp. son escasos.

Entre las herbáceas el grupo con mayor porcentaje es el de Poaceae (74\%), Triglochin sp. (Juncaginaceae) con un máximo de 39\%, seguidas por Baccharis sp. (16\%), periporados del Tipo Chenopodium (13\%), Bidens sp. (Asteraceae) (10\%), el Tipo Amaranthus y Atriplex sp. con el $9 \%$ respectivamente. Por otra parte, el Tipo Gomphrena (2-7\%), y Senecio sp. (12\%) están presentes solamente en algunos niveles y Eryngium campestre L., escasamente representado en la base y final de esta zona con el 4\%. Entre los indicadores de humedad local se encuentran Cyperus giganteus Vahl (15$20 \%$ ) y el complejo Typhaceae-Sparganiaceae que alcanza el 3\%. Las pteridófitas Microgramma vacciniifolia Langsd. y Fisch y Asplenium sp. son las más abundantes con el $10 \%$ y $6 \%$ respectivamente, continúa Trichomanes sp. (2-4\%). Sphagnum sp. varía de 2 al $12 \%$. Las algas Botrioccocus sp. (70\%) y Spirogyra sp. (9\%) son las más numerosas, no obstante, Pediastrum sp. está presente en bajos porcentajes $(<3 \%)$. Los valores de concentración polínica varían en la zona desde 224 413granos/g hasta 449 066granos/g, alcanzando su máximo a los $14 \mathrm{~cm}$ de profundidad.

Zona B: Desde los $10 \mathrm{~cm}$ de profundidad hasta la superficie. Está caracterizada por la disminución en el porcentaje de la mayoría de las especies arbóreas, con la excepción de Tecoma stans y Prosopis sp., que alcanzan el $4 \%$. En el estrato herbáceo se observan los mayores porcentajes de toda la columna sedimentaria de Bidens sp. (12\%) y Baccharis sp. (17\%), también se observa la disminución de Poaceae (40\%), Triglochin sp. (30\%), los periporados Tipo Amaranthus (5\%,), Tipo Chenopodium (9\%), Senecio sp. (10\%) y Apocynaceae $(<1 \%)$. Aparece Eryngium campestre cuyo porcentaje se mantiene constante en $7 \%$. Entre los indicadores de humedad local se acentúa la presencia de Cyperus giganteus (20-24\%), mientras que disminuye el Complejo Typhaceae-Sparganiaceae del $2 \%$ al $0,5 \%$. 


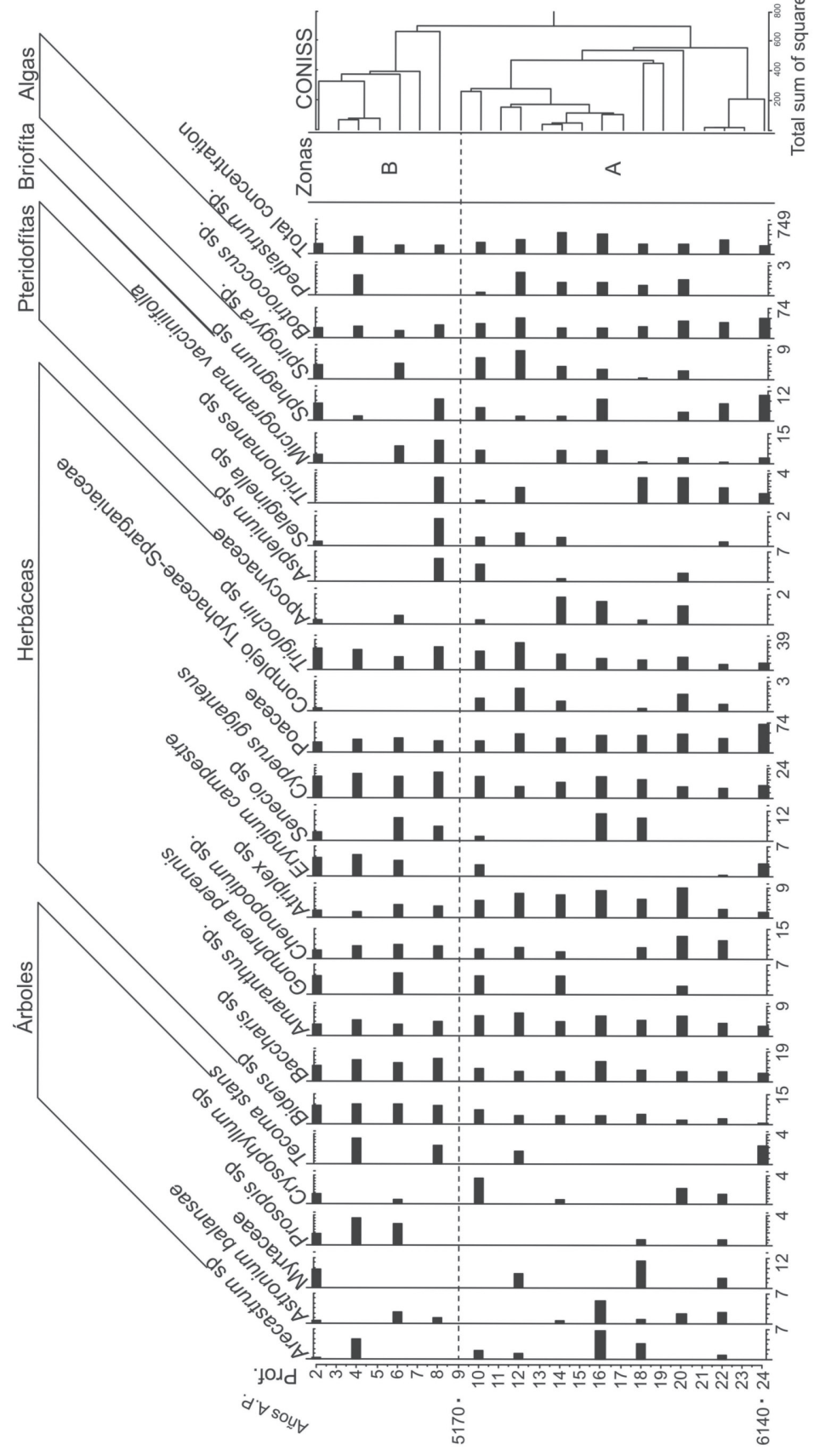

Fig. 3. Diagrama polínico porcentual de la laguna San Sebastián, incluye dataciones radiocarbónicas y concentración polínica total (granos/g).

Fig. 3. Porcentaje palynological diagram of the lagoon San Sebastián, includes radiocarbon data and palynological total concentration (grains/g). 


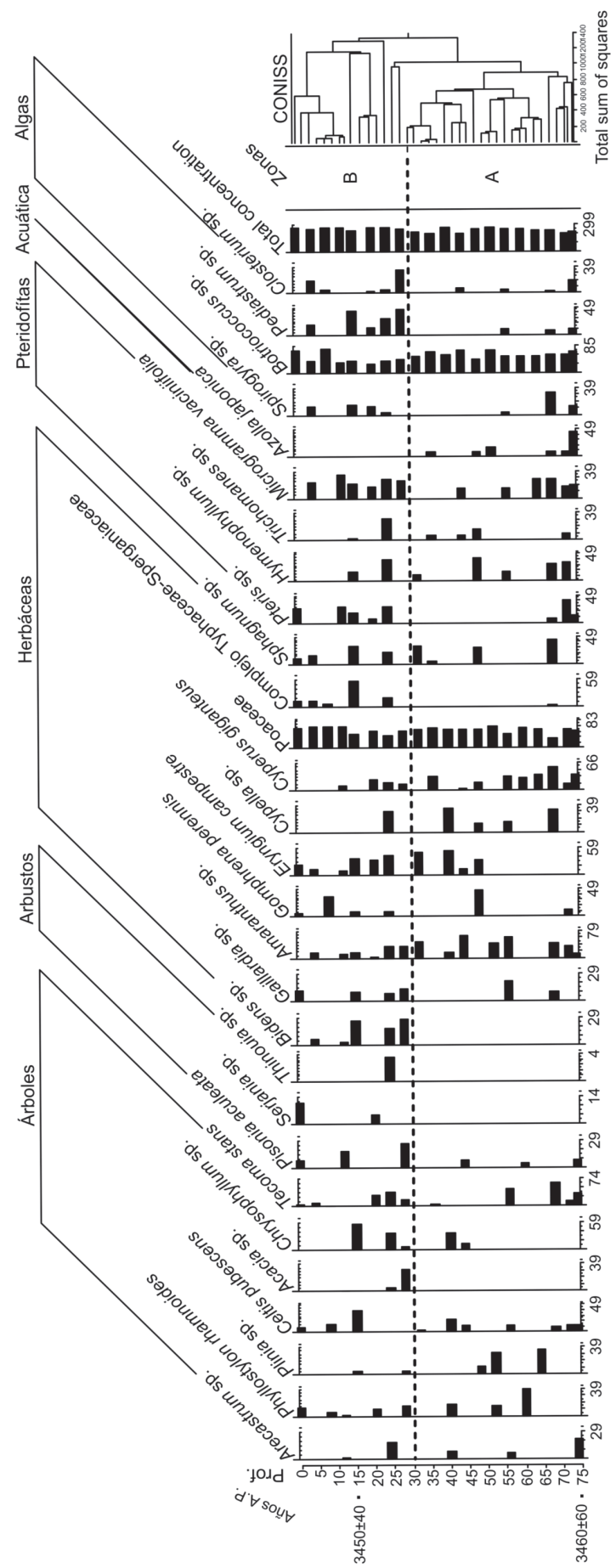

Fig. 4. Diagrama polínico porcentual de la laguna San Juan, incluye dataciones radiocarbónicas y concentración polínica total (granos/g). (Modificado de Arbo \& Tressens 2002).

Fig. 4. Pollen percentage diagram of Lake San Juan, including radiocarbon and total pollen concentration (grains/g). (Modified from Arbo \& Tressens 2002). 
Las pteridófitas también disminuyen y luego desaparece su representación desde la base hacia los niveles superficiales de esta zona. Las algas Botrioccocus sp. (70-30\%) y Spirogyra sp. (9-5\%) también disminuyen en esta zona, mientras que Pedistrum sp. se encuentra representado solamente en el nivel 4 con el $2 \%$. La concentración polínica total aumenta de 149 891granos/g a los $8 \mathrm{~cm}$ de profundidad a 299 733granos/g a los $4 \mathrm{~cm}$ de profundidad, alcanzando aquí la máxima concentración en esta zona.

Laguna San Juan: Se diferencian 2 zonas (A y B) (Fig. 4)

Zona A: Desde los 75 a los $35 \mathrm{~cm}$ de profundidad. El aporte polínico arbóreo está dado por el registro de Arecastrum sp. (20\%), Myrtaceae del género Plinia Plum. ex L. (30\%), Celtis pubescens (Humb. y Bonpl.) Spreng. (20\%), Phyllostylon rhamnoides (J.Poiss.) Taub. (39\%), Chrysophyllum sp. (40\%) y Tecoma stans (60\%). Pisonia aculeata Kuntze (10\%) es el único representante de los arbustos en la zona. Integrando el estrato herbáceo predominan las poáceas con porcentajes elevados y relativamente estables $(70 \%)$, periporados del Tipo Amaranthus (60\%) y Erymgium campestre con el (50\%) que se registra por primera vez a los $50 \mathrm{~cm}$ de profundidad. Entre los taxa que señalan humedad local se hallan, Cypella sp. (Iridaceae) con el 34\%, y las ciperáceas aunque decrece su porcentaje hasta el $59 \%$. La vegetación acuática está representada por Azolla japonica Franch. y Sav. (44\%) y las algas: Botrioccocus sp. (70\%); Spirogyra sp. (32\%), Closterium sp. (19\%) y Pediastrum sp. (10\%). Esta última sólo está presente en los niveles inferiores de la zona. La concentración polínica total disminuye de 261 625granos/g en los niveles basales de la zona a $186875 \mathrm{granos} / \mathrm{g}$ en los niveles superiores.

Zona B: Desde los $35 \mathrm{~cm}$ hasta la superficie. En el registro del polen arbóreo se distingue: el aumento de Chrysophyllum sp. (59\%) y Celtis pubescens (44\%), la disminución de Arecastrum sp. (16\%), Phyllostylon rhamnoides (12\%), Tecoma stans (45\%) y la aparición de Acacia sp. (34\%). Entre los representantes del estrato arbustivo predominan Serjania sp. con el $12 \%$, Pisonia aculeata (27\%) y Thinouia sp. Springer (4\%). En el estrato herbáceo se encuentran en orden de abundancia las poáceas (66\%), el complejo Typhaceae-Sparganiaceae (56\%), el Tipo Gomphrena (37\%), Eryngium campestre (44\%), el Tipo Amaranthus (41\%), Bidens sp. (29\%) y Gaillardia sp. (13\%). Botriococcus sp. alcanzó el máximo porcentaje de toda la columna sedimentaria $(85 \%)$ seguido de Pediastrum sp. con (45\%), otras algas mucilaginosas con $(42 \%)$ y Closterium sp. (35\%). La concentración polínica total aumenta a 261 625 granos/g y se mantiene relativamente estable a lo largo de la zona.

Para interpretar la secuencia y cambios de la vegetación en el área (Fig. 5), se relacionaron los perfiles de ambas lagunas por su edad, siendo San Sebastián la de mayor edad.

\section{DISCUSIÓN}

El análisis palinológico de ambas lagunas permite distinguir, para el NO del Iberá, los siguientes cambios vegetacionales: desde los

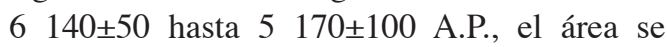
caracterizó por una vegetación predominantemente herbácea, compuesta principalmente por Poaceae, Baccharis sp., Complejo TyphaceaeSparganiaceae y Triglochin sp., frecuentes en ambientes palustres. El polen proveniente de la vegetación arbórea integrada por Arecastrum sp., Astronium balansae y Tecoma stans está escasamente representada, probablemente debido a que su aporte provenía de zonas mas alejadas al cuerpo de agua, integrando la vegetación regional, estas especies forman actualmente una vegetación abierta características de ambientes secos.

El predominio de la vegetación palustreherbácea concuerda con lo publicado por Iriondo (1994) quien señala que, durante el Holoceno Inferior y Medio en el norte de Argentina y principalmente en áreas próximas al río Paraná (en la provincia del Chaco), la acción de la erosión retrocedente formó redes fluviales, que se 


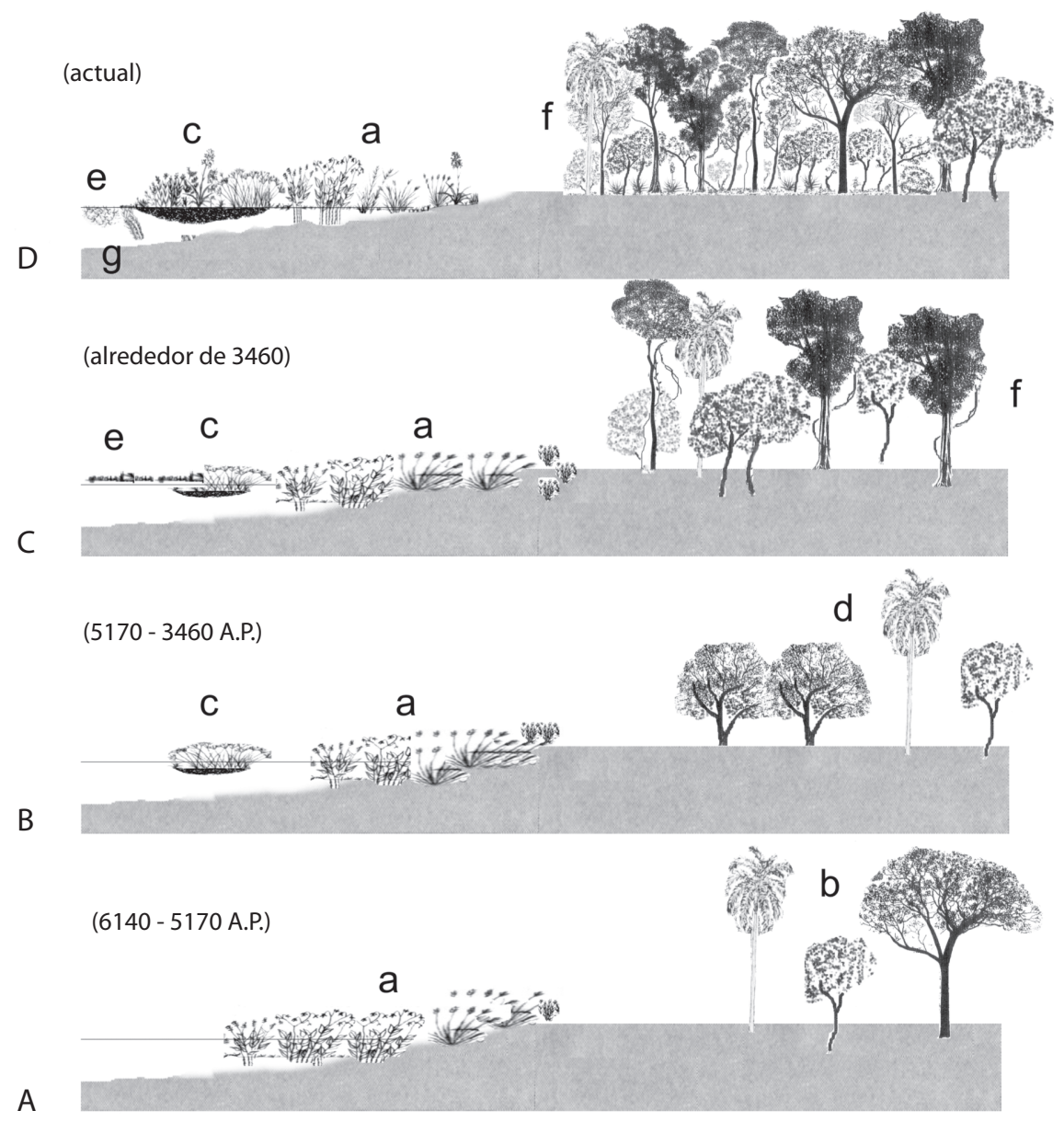

Fig. 5. Perfiles esquemáticos de la vegetación desde los 6140 años A.P. hasta la actualidad, mostrando: A. (a) vegetación palustre-herbácea; (b) vegetación arbórea abierta. B. (c) embalsado; (a) vegetación palustre-herbácea; (d) bosque de Prosopis. C. (e) macrófitas flotantes; (c) embalsado; (a) vegetación palustre-herbácea; (f) bosque higrófilo. D. (g) macrófitas sumergidas; (e) macrófitas flotantes; (c) embalsado; (a) vegetación palustre-herbácea; (f) bosque higrófilo.

Fig. 5. Schematic profiles of vegetation from 6140 years a.C. to present, showing: A. (a) herbaceous marsh vegetation; (b) open tree vegetation. B. (c) embalsado; (a) herbaceous marsh vegetation; (d) Prosopis`s forest. C. (e) floating macrophytes; (c) embalsado; (a) herbaceous marsh vegetation; (f) riverfront forests. D. (g) submerged macrophytes; (e) floating macrophytes; (c) embalsado; (a) herbaceous marsh vegetation; (f) riverfront forests.

sabe estaban bien desarrolladas por la presencia de sedimentos palustres.

Desde los 5 170 \pm 100 en adelante, la frecuencia y variedad de especies características de ambientes húmedos disminuyó, no obstante, continuó el predominio de las Poaceae; también estaban representadas las Asteraceae
(Baccharis sp., Bidens sp. etc.) y Eryngium campestre, especies colonizadoras de espejos de los cuerpos de agua. Esta asociación polínica, junto a la disminución porcentual de las algas (Clorofíceas), sugieren el desarrollo de embalsados, probablemente como consecuencia de una mayor colmatación de la laguna, 
ocasionando reducción del espejo de agua, mayor oscuridad, déficit de oxígeno y acidez del agua.

El polen proveniente de la vegetación arbórea circundante muestra un aumento del aporte polínico arbóreo, esto junto a la aparición continua de Prosopis sp. y el aumento de la concentración polínica, que sugiere mayor desarrollo de una vegetación arbórea característica de ambientes más secos, en donde Prosopis $\mathrm{sp}$. fue la especie dominante.

Alrededor de los 3500 años A.P. para el Norte de la provincia de Santa Fe (al NO del presente sitio de estudio), Garralla (1998) destacó también el predominio de vegetación de bosque xerohalófilo y el desarrollo de condiciones climáticas secas, lo que concuerda con la composición vegetal descrita.

Desde los $3460 \pm 60$ A.P. en adelante, continuó el predominio de la vegetación palustreherbácea, pero con ligeras variantes en su composición vegetal. En una primera etapa la vegetación herbácea, se encontraba integrada principalmente por Poaceae, Amaranthus sp. y Cyperaceae característicos de ambientes palustres. El aporte polínico proveniente de la vegetación acuática, indica que el cuerpo de agua se encontraba colonizado por macrófitos (Azolla). Con respecto a los taxones arbóreos (Phyllostylon rhamnoides, Plinia sp., Tecoma stans, Acacia sp., Celtis pubescens, Arecastrum sp. y Chrysophyllum sp.), que hoy son frecuentes en lomas de arenas rojas y amarillentas, próxima a esteros, lagunas, bañados y arroyos, sugieren el comienzo del desarrollo de un bosque higrófilo.

En una etapa posterior, se observa el incremento en porcentaje y variabilidad de especies palustres-herbáceas. Por otra parte, si bien continuó el predominio del bosque higrófilo, este se vió enriquecido por el incremento en el número de especies arbóreas y arbustivas, pues aparecen registrados Acacia sp., Serjania sp. y Thinouia sp.; además aumentan su representación Pisonea aculeata, Chrysophyllum sp. y Celtis pubescens, y disminuyen Phyllostylon rhamnoides, Tecoma stans y Arecastrum sp.
Por último, es de destacar, que, las diferencias encontradas entre la cantidad de sedimento depositado en el fondo de las lagunas analizadas y el tiempo transcurrido, puede relacionarse con la ubicación geomorfológica de las lagunas en la región; la laguna San Sebastián habría estado situada en una lomada y por ello recibiría menor aporte sedimentario; mientras que la laguna San Juan habría estado emplazada en una depresión, recibiendo en consecuencia mayor aporte. La presencia de lomadas en la región de estudio, se debe a que en el área de los esteros del Iberá, la Formación Ituzaingó yace en discordancia erosiva sobre los basaltos y/o areniscas del Grupo Solari-Serra Geral que aflora en superficie en forma de abanico formando cordones discontinuos de distribución radial con ápice en el área de Ituzaingó. Estas lomadas cruzan diagonalmente la mitad noroccidental de la provincia dejando evidencias de importantes de corrientes fluviales que atravesaron el territorio actualmente ocupado por los esteros del Iberá (Orfeo 2005)Cabe concluir que en el área de estudio, alrededor de 6000 años A.P., predominó la vegetación herbácea en un clima húmedo, este período húmedo finalizó hacia los 5200 años A.P. tendiendo a condiciones climáticas más secas ya que entre 5170 años A.P. y 3500 años A.P. aproximadamente se establece la vegetación de bosque xerohalófilo y la colmatación del espejo de agua, la aparición de embalsados. A partir de los 3500 años A.P. la frecuencia y variedad de especies características de ambientes húmedos aumenta, predominando las especies palustres-herbáceas, además del desarrollo del bosque higrófilo, antecesores de las asociaciones actuales.

\section{RESUMEN}

Los humedales son sitios de gran importancia para los estudios palinológicos, ya que representan uno de los ambientes más idóneos para la preservación del polen fósil. El objetivo del trabajo es determinar, mediante del análisis palinológico de sedimentos lacustres, las comunidades vegetales y el ambiente predominante durante el Holoceno en el NO del Iberá, ya que los humedales representan uno de los ambientes más aptos para la preservación del polen 
fósil. Las lagunas estudiadas son: San Sebastián y San Juan Poriahú, cuyos sedimentos fueron obtenidos con un sacatestigo "Levingstone square-rod sampler", las muestras fueron procesadas con las técnicas de Faegri e Iversen y datadas con $\mathrm{C}^{14}$. Los diagramas palinológicos se dividieron en zonas utilizando el programa Tilia. El análisis palinológico permite distinguir diversos cambios vegetacionales:

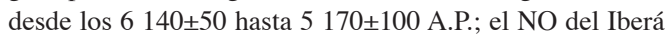
se caracterizó por una vegetación palustre-herbácea y una vegetación arbórea característica de ambientes secos. Desde los $5170 \pm 100$ hasta $3460 \pm 60$ A.P., se produce la disminución en la frecuencia de especies características de ambientes húmedos y la colmatación del cuerpo de agua. Desde los $3460 \pm 60$ A.P. en adelante, si bien continúa el predominio de la vegetación herbácea característica de ambientes palustres, el polen arbóreo, indica el comienzo del desarrollo de un bosque higrófilo.

Palabras clave: humedales, palinomorfos, sedimentos lacustres, comunidades vegetales, ambiente.

\section{REFERENCIAS}

Arbo, M.M. \& S.G Tressens. 2002. Flora de Iberá. EUDENE, Corrientes, Argentina.

Canevari, P., D. Blanco, E. Bucher, E. Castro \& I. Davidson. 1998. Los humedales de la Argentina: clasificación, situación actual, conservación y legislación. Wetlands International, Publicación No. 46. Secretaría de Recursos Naturales y Desarrollo Sustentable, Buenos Aires, Argentina.

Carnevali, R. 1994. Fitogeografía de la Provincia de Corrientes. Gobierno de la Provincia de Corrientes-Instituto Nacional de Tecnología Agropecuaria. Corrientes, Argentina.

Cuadrado, G.A. \& J.J. Neiff. 1993. Palynology of embalsados in distrophic lakes in Northeastern of Argentina. Rev. Bras. Biol. 53: 443-451.

Faegri, K. \& J. Iversen. 1989. Textbook of Pollen Analysis. Wiley, Nueva York, EEUU.

Fernández Pacella, L.E. 2005. Aportes al conocimiento de la Paleodiversidad del Cuaternario del Iberá. Reunión de Comunicaciones, SECYT-UNNE. Corrientes, Argentina.

Garralla, S. 1998. Estudio palinológico de una secuencia sedimentaria del Holoceno, Norte de Santa Fe, Argentina. Polen 9: 17-27.

Iriondo, M. 1981. Antigüedad del último cambio climático en el Litoral. Ecol. Arg. 6: 5-8.

Iriondo, M. 1994 Los climas cuaternarios de la región Pampeana. Comunicaciones del Museo Provincial de Ciencias Naturales "Florentino Ameghino" 4: 481.

Neiff, J.J. 1977. Investigaciones ecológicas en el complejo de la laguna Iberá en relación a diversas formas de aprovechamiento hídrico. Seminario sobre Medio Ambiente y Represas, 70-87. Montevideo, Uruguay.

Neiff, J.J. 1981 Panorama ecológico de los cuerpos de agua del nordeste argentino. Simposio. IV Jornadas Argentina de Zoología: 115-151.

Neiff, J.J. 1981b. Vegetación acuática y anfibia del Iberá, Corrientes. II: Dinámica, p 86-165. In Investigaciones Ecológicas en el Macrosistema Iberá. ICACECOAL, Informe Final.

Neiff, J.J. 1982. Esquema sucesional de la vegetación en islas flotantes del Chaco Argentino. Boletín de la Sociedad Argentina de Botánica 21: 325-341.

Neiff, J.J. 1997. Ecología evolutiva del macrosistema Iberá (Corrientes, Argentina).Tesis de Maestría, Universidad Nacional del Litoral, Santa Fe, Argentina.

Orfeo, O. 2005. Historia Geológica del Iberá, provincia de Corrientes, como escenario de biodiversidad. Miscelánea INSUGEO 14: 71-78.

Poi de Neiff, A. 1981. Fauna asociada a la vegetación acuática y palustre del Iberá, p. 166-181. In Investigaciones ecológicas en el macrosistema Iberá. Informe convenio Instituto Correntino del Agua y Centro de Ecología Aplicada del Litoral, Corrientes.

Popolizio, E. 1970. Algunos rasgos de la geomorfología del nordeste argentino. Boletín de la Sociedad Argentina Botánica 11: 17-34. 